\title{
Volvulus of the Sigmoid Colon Associated With Rectal Cancer: A Case Report
}

\author{
Seung-Hyun Lee ${ }^{\mathrm{a}, \mathrm{b}}$, Byung-Kwon Ahn ${ }^{\mathrm{a}}$, Sung-Uhn Baek ${ }^{\mathrm{a}}$
}

\begin{abstract}
Sigmoid volvulus is one of the three most common causes of acute colonic obstruction. Predisposing factors include chronic constipation, adhesion from a prior abdominal surgery, and megacolon. However, concomitant presentation of volvulus of the sigmoid colon and rectal cancer is extremely rare. We report a case of a 50-year-old woman with coexisting volvulus of the sigmoid colon and rectal cancer. The patient presented with abdominal distension and pain for 2 days. On computed tomography, the whole colon was dilated with gas and feces. A whirl sign with rotation of the inferior mesenteric vessel was identified. The rectum had irregular wall thickening. Colonoscopy showed a circumscribed, ulcerofungating mass approximately $6 \mathrm{~cm}$ from the anal verge. The sigmoid colon was obstructed at a point approximately $25 \mathrm{~cm}$ from the anal verge. The mucosa was hyperemic and edematous with the pathognomonic spiral pattern. Endoscopic reduction was not successful. On laparotomy, the sigmoid colon was rotated around its mesentery. It was severely distended with edematous, hyperemic serosa. A tumor of the rectum was identified in the mid-rectum. The patient underwent low anterior resection and protective ileostomy. Pathologic findings confirmed adenocarcinoma of the rectum. The postoperative course was complicated by an ileus, which was managed with conservative treatment.
\end{abstract}

Keywords: Intestinal volvulus; Sigmoid disease; Neoplasms; Rectum

\section{Introduction}

Sigmoid volvulus is one of the three most common causes of acute colonic obstruction [1]. It refers to the twisting or torsion of the sigmoid colon about its mesentery. The etiology of this

Manuscript accepted for publication August 24, 2014

${ }^{a}$ Department of Surgery Kosin University College of Medicine, Busan, Korea ${ }^{b}$ Corresponding Author: Seung-Hyun Lee, Department of Surgery, Kosin University College of Medicine, 262 Gamcheon-ro, Seo-gu, Busan 602-702, Korea. Email: gscrslsh@hanmail.net

doi: http://dx.doi.org/10.14740/gr619w disorder is not completely understood. It is known to occur in the setting of a redundant sigmoid loop, which rotates around its narrow and elongated mesentery [2]. Predisposing factors include chronic constipation, adhesion from a prior abdominal surgery, and megacolon. However, presentation as coexisting sigmoid volvulus and rectal cancer is extremely rare. We report a case of concomitant volvulus of the sigmoid colon and rectal cancer.

\section{Case Report}

A 50-year-old woman presented to the emergency department with lower abdominal pain and distension. Several months prior to admission, she experienced a change in her bowel habits with intermittent constipation and diarrhea. For 2 months, she also experienced intermittent bloody stool with a change in her stool caliber. Approximately $4-5 \mathrm{~h}$ prior to presentation, she experienced acute, severe lower abdominal pain. She did not complain of nausea and vomiting. She had a past surgical history of a cesarean section approximately 20 years ago.

On admission, the patient's height, weight, and body mass index were $145 \mathrm{~cm}, 40 \mathrm{~kg}$, and 19.0, respectively. She had a weight loss of approximately $10 \mathrm{~kg}$ over the past year. Vital signs were normal with a blood pressure of $110 / 80 \mathrm{~mm} \mathrm{Hg}$, pulse rate of 52 beats $/ \mathrm{min}$, respiration rate of $20 / \mathrm{min}$, and body temperature of $36.7^{\circ} \mathrm{C}$. Physical examination revealed tenderness and rigidity in the left lower abdominal quadrant. Intestinal sounds were strongly audible with increased frequency on auscultation. Laboratory investigation revealed the following: white blood cell count, $7,510 / \mathrm{mm}^{3}$ (63.9\% neutrophils); hemoglobin, $9.9 \mathrm{~g} / \mathrm{dL}$; hematocrit, $32.1 \%$; platelet count, $323 \times$ $10^{3} / \mathrm{mm}^{3}$; serum protein, $6.5 \mathrm{~g} / \mathrm{dL}$; serum albumin $3.7 \mathrm{~g} / \mathrm{dL}$; total bilirubin, $0.36 \mathrm{mg} / \mathrm{dL}$; alanine aminotransferase, $11 \mathrm{IU} / \mathrm{L}$; aspartate aminotransferase, $7 \mathrm{IU} / \mathrm{L}$; alkaline phosphatase, 79 U/L; amylase, $46 \mathrm{U} / \mathrm{L}$; lipase, $19 \mathrm{U} / \mathrm{L}$; creatinine, $0.58 \mathrm{mg} / \mathrm{dL}$; serum Na, $134 \mathrm{mEq} / \mathrm{L}$; serum $\mathrm{K}, 3.2 \mathrm{mEq} / \mathrm{L}$; and carcinoembryonic antigen, $1.70 \mathrm{ng} / \mathrm{mL}$.

On computed tomography, the whole colon was dilated with gas and feces. A whirl sign with rotation of the inferior mesenteric vessel was identified (Fig. 1a). The rectum had irregular wall thickening (Fig. 1b). Colonoscopy showed a circumscribed, ulcerofungating mass approximately $6 \mathrm{~cm}$ from the anal verge. The sigmoid colon was obstructed at a point 


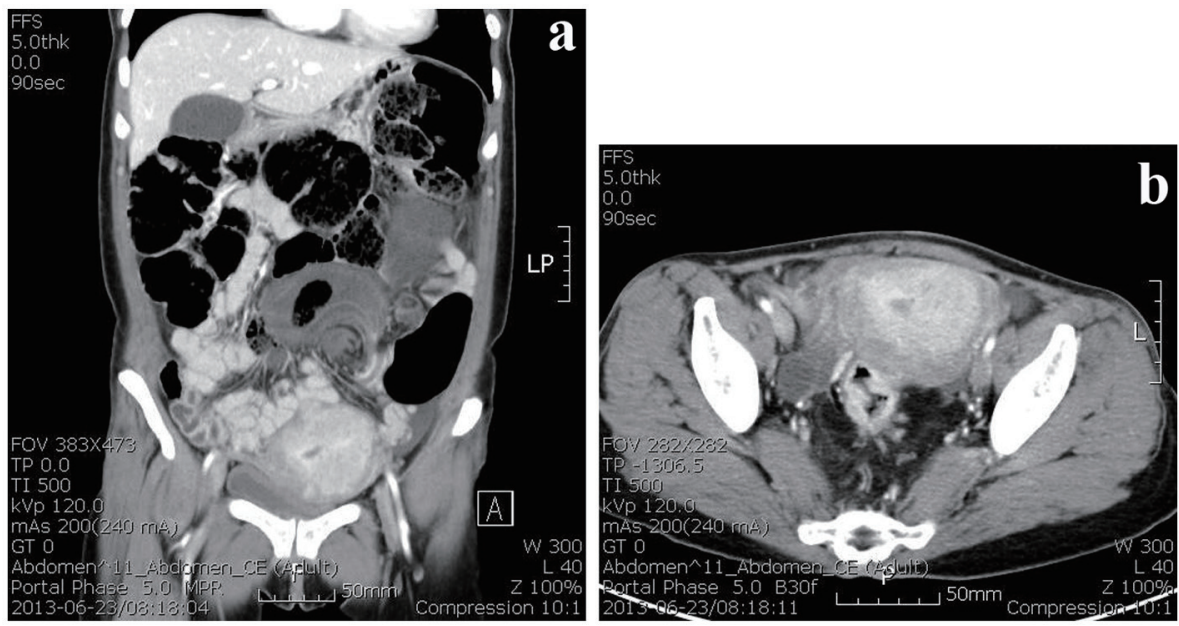

Figure 1. CT findings show whirl sign with rotation of inferior mesenteric vessel was identified (a). The rectum had irregular wall thickening (b).

approximately $25 \mathrm{~cm}$ from the anal verge. The mucosa was hyperemic and edematous with the pathognomonic spiral pattern of a volvulus. Endoscopic reduction was not successful.

On laparotomy, the sigmoid colon was rotated around its mesentery approximately $270^{\circ}$. It showed severe dilatation with edematous, hyperemic serosa (Fig. 2). A tumor of the rectum was identified in the mid-rectum. The remainder of the large bowel was unremarkable. The patient underwent low anterior resection and protective ileostomy. Pathologic findings confirmed adenocarcinoma of the rectum. On pathologic

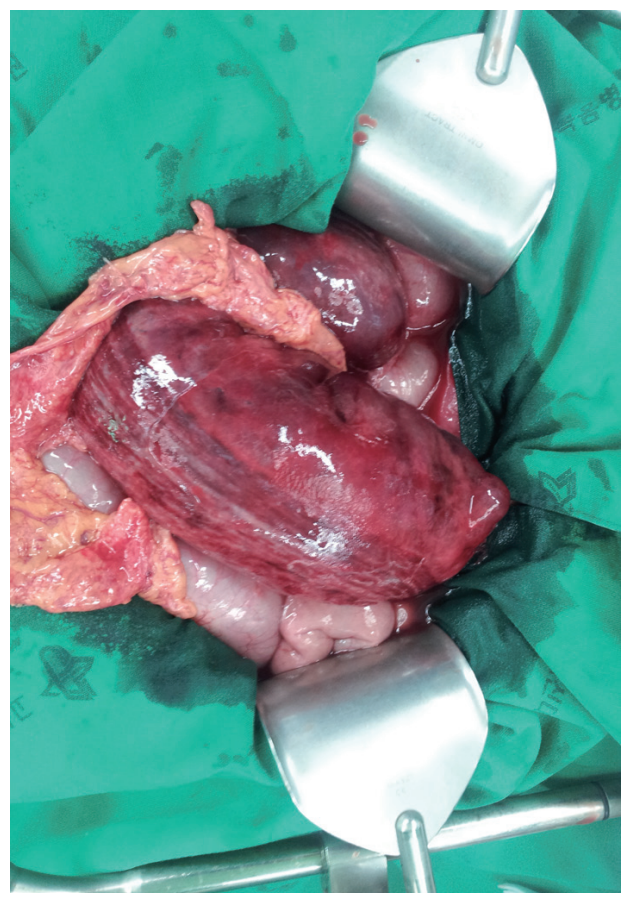

Figure 2. Operative finding shows the sigmoid colon rotation around its mesentery about $270^{\circ}$. It has severely dilatation with edematous, hyperemic serosa. examination, the tumor invaded to the pericolic fat tissue. The tumor was identified in four lymph nodes out of a total of 21 sampled lymph nodes. The postoperative course was complicated by an ileus, which was managed with conservative treatment.

\section{Discussion}

Sigmoid volvulus refers to the twisting or torsion of the sigmoid colon about its mesentery. It comprises $50-90 \%$ of all cases of colonic volvulus [1]. The etiology of this disorder is not completely understood. A long redundant sigmoid colon with a narrow mesentery is a predisposing condition for sigmoid volvulus formation. Chronic fecal overloading is believed to cause elongation and dilatation of the sigmoid colon [2]. Chronic fecal overloading is related to chronic constipation and adhesion from a prior abdominal surgery. A coexistent colon tumor may be considered as a predisposing factor for colon volvulus, which is associated with aggravating proximal bowel dilatation. In this report, the patient had two predisposing factors, including a medical history of previous abdominal surgery and a concurrent colon tumor. Operative findings showed no significant intra-abdominal adhesions, which can be a cause of chronic fecal overloading.

The presentation of colon volvulus with a coexistent tumor of the colon is extremely rare. Lapin et al [3] reported a case of a 36-year-old woman with a volvulus of the transverse colon associated with a submucosal hamartoma. The patient had chronic constipation with a family history of Von Recklinghausen's disease. The submucosal hamartoma was found in a resection specimen of the transverse colon. Meyers et al [4] reported a case of a 54-year-old man with a cecal volvulus and an obstructive carcinoma of the left colon. Figiel and Figiel [5] reported a case of an 80-year-old woman with ascending colon volvulus and a coexistent carcinoma at the splenic flexure. Wecksell and Gordon [6] reported a case of an 80 -year-old man with an ascending colon volvulus associated 
with an adenocarcinoma of the transverse colon. Natarajan et al [7] reported a case of a 70-year-old woman with a cecal volvulus and a coexistent carcinoma at the rectosigmoid colon. The last three cases were cecal or ascending colon volvulus with an age range of $70-80$ years old. This is the first case report of a sigmoid volvulus with concurrent rectal tumor.

Based on our case review, it is important for the surgeon to examine the entire colon when operating on cases of colon volvulus to identify concurrent colon tumors.

\section{References}

1. Ballantyne GH, Brandner MD, Beart RW, Jr., Ilstrup DM. Volvulus of the colon. Incidence and mortality. Ann Surg. 1985;202(1):83-92.
2. Margolin DA, Whitlow CB. The pathogenesis and etiology of colonic volvulus. Semin Colon Rectal Surg. 2007;18:79-86.

3. Lapin R, Kane AA, Lee CS, Rifat H. Volvulus of the transverse colon associated with submucosal hamartomas. Am J Gastroenterol. 1973;59(2):170-173.

4. Meyers JR, Heifetz CJ, Baue AE. Cecal volvulus: a lesion requiring resection. Arch Surg. 1972;104(4):594-599.

5. Figiel LS, Figiel SJ. Volvulus of the cecum and ascending colon. Radiology. 1953;61(4):496-515.

6. Wecksell A, Gordon LA. Simultaneous double obstruction of the proximal colon: a case report. Gastrointest Radiol. 1979;4(3):303-305.

7. Natarajan A, D'Souza RE, Lahoti NG. A rare case of dual obstruction of the colon. Trop Gastroenterol. $2001 ; 22(4): 215-216$. 